\section{Modeling of Conducted Common Mode Perturbations in Variable-Speed Drive Systems}

\author{
François Costa, Christian Vollaire, and Régis Meuret
}

\begin{abstract}
In this paper, common mode (CM) conducted perturbations are predicted and compared with experiments in a variable-speed drive system, thanks to a mixed approach based on experimental measurements and on the modeling of the complete $\mathrm{CM}$ circuit. Its different parts are considered and represented by a chain of quadripolar matrices: the inverter, the cables, and the induction motor. At last, it is shown that the parasitic currents in the system can be calculated in the different stages of the matrix chain. Experiments have successfully confirmed this approach.
\end{abstract}

Index Terms-Conducted perturbations, quadripolar matrices modeling, variable-speed drive.

\section{INTRODUCTION}

Voltage inverters used in a variable-speed drive are major sources of common mode $(\mathrm{CM})$ disturbances leading to multiple consequences: parasitic currents, radiation of cables, destruction of motor's bearings, overvoltage, and conducted emissions in long cables [1]-[4], [8]. Consequently, it is necessary to characterize as accurately as possible these

Manuscript received July 2, 2004; revised May 30, 2005.

F. Costa is with SATIE UMR 8029, 94230 Cachan, France (e-mail: francois.costa@satie.ens-cachan.fr).

C. Vollaire is with CEGELY, Ecole Centrale de Lyon, 69134 Ecully, France (e-mail: vollaire@ec-lyon.fr).

R. Meuret is with society SAFRAN, group Hispano-Suiza, 77550 Moissy Cramayel, France (e-mail: regis.meuret@hispano-suiza-sa.com).

Digital Object Identifier 10.1109/TEMC.2005.857365

\section{EXPERIMENTAL BENCH}

The experimental bench has been built to meet with normative measurements as indicated in the European standard EN 55011. It is designed to represent a typical variable-speed drive system: it includes a front-end, three-phase transformer $(400 \mathrm{VAV} / 50 \mathrm{~Hz})$ and an insulated grid bipolar transistor (IGBT) inverter that feeds the induction motor through long shielded cables. The mechanical load is constituted by a dc generator loaded by a bulk resistor. Fig. 1 shows a picture of the electrical machines.

The complete system is situated on a $2-\mathrm{m}^{2}$ copper plane electrically connected to ground. The dc generator and the induction motor are placed on a thick PVC plate to insulate them from the ground. The motor bearings have been insulated with PVC rings to separate parasitic current flowing from the stator and from the rotor. The linking between the motor and its load can also be insulated, the same for the linking between the motor and its driving sensor. Every part of the system can be linked separately to ground so as to estimate its own contribution to the $\mathrm{CM}$ current. Thus, it is possible to measure the parasitic $\mathrm{CM}$ current issued from any part of the full system as presented in the schematic representation of Fig. 2 to compare them with simulation or to estimate the dominant effects.

This experimental bench elements are depicted here:

- three-phase transformer: $400 \mathrm{~V}, 50 \mathrm{~Hz}, 4 \mathrm{kVA}$;

- line impedance stabilized network (LISN): $50 \Omega 4 \times 15 \mathrm{~A}-$ MN2053, Chase Electronics Ltd.;

- variable-speed drive: Schneider Electric ATV 58. It is constituted by an IGBT module (SEMIKRON), and its switching frequency is adjustable from 2 to $16 \mathrm{kHz}$;

- induction motor (Leroy Somer), $3 \times 400 \mathrm{~V} / 3 \mathrm{~kW}$, nominal speed: $1500 \mathrm{r} / \mathrm{min}$ 


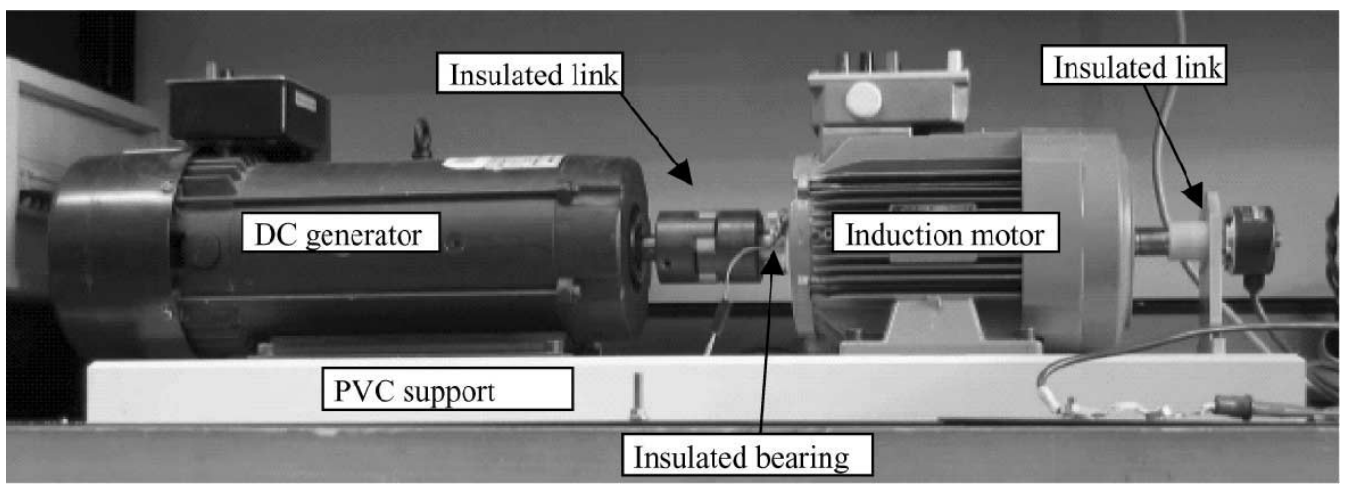

Fig. 1. CM bench.

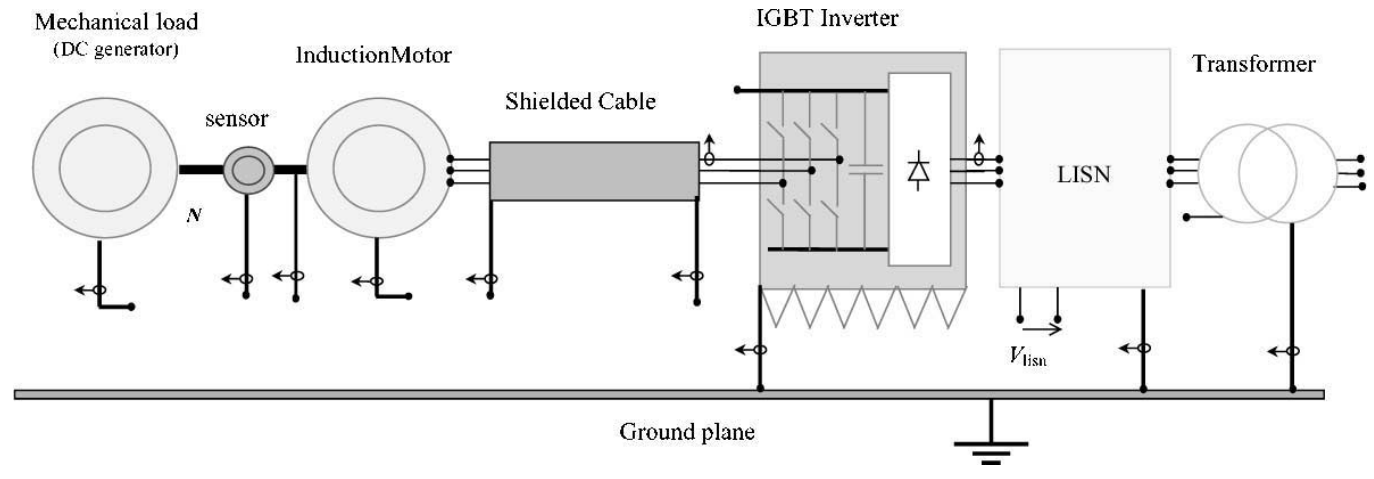

Fig. 2. Schematic representation of the complete system.

- numerical encoder: Heidenhain ERN 420;

- shielded cables: four conductors, section $=5 \mathrm{~mm}^{2}+$ shielding braid.

$\mathrm{CM}$ currents in this kind of system result mainly from two origins: 1) the $\mathrm{CM}$ voltage of an inverter is never null because of the modulation principle: it shows large and fast variations at the switching frequency, depending on the pulsewidth modulation (PWM) strategy, this point is highlighted later in the article; and 2) capacitive couplings to ground are distributed in the whole system, which create multiple CM circuits: within the converter, in the cable shielding, and in the motor.

Fig. 3 shows a simplified lumped element representation of the system. $Z_{\text {LISN }}$ represents the impedances of the LISN between the dc bus and the ground constituted by the LISN. However, even if there is no LISN, these impedances exist because of all parasitic capacitances in the main or in the front-end transformer. The capacitances $C_{\text {inv }}$ are representative of all capacitive couplings between the power semiconductors and ground (i.e., semiconductor case to heat-sink capacitances, printed circuit board (PCB) layout capacitances, and IGBT drivers to ground capacitances). In our experimental bench, the value of $C_{\mathrm{inv}}$ is in the order of $300 \mathrm{pF}$ per inverter leg. In this equivalent circuit, the induction motor is characterized by its winding impedance $Z_{\mathrm{w}}$ and by its CM impedance $Z_{\mathrm{t}}$. The weigh of each CM coupling of the system is linked to various parameters as the inverter technology, the length of the cables and their position relative to ground, the presence of a shielding, its ends connections, and finally, the manufacturing of the motor.

\section{MODELING}

\section{A. Modeling Description}

As previously explained, the numerical simulation of such a system would be quite complicated, requiring three-dimensional simula- tion tools for electromagnetic effects (in cables, motor, inverter); then accurate lumped models would have to be fed by other tools before a "circuit" simulation tool could be used to calculate parasitic currents in the system. This kind of simulation process is not well matched to the EMI analysis over a large frequency range and leads to long simulation time for a likely mediocre accuracy. That is the reason why we have adopted an intermediate approach to predict conducted currents. First, we have decided to work in frequency domain because EMI analysis is generally done in this way. So, EMI sources are identified and acquired in the form of a spectrum. In this way, all harmonic components of the signal are known and can be used for calculations. Second, we have adopted a mixed approach to model the CM circuit of the system, based on experimental characterization of the impedances and on model association. The CM equivalent circuit of the system is represented by a chain of quadripolar matrix, each corresponding to a part of the system. Third, we have constituted experimental database of these elements in the form of quadripolar impedance matrices. Fig. 4 shows the model corresponding to the system of Fig. 3. The CM voltage $V_{\mathrm{cm}}$ due to the inverter is the main CM source of the system.

\section{B. Modeling Hypothesis}

Our modeling principle is based on the idea that the CM currents are essentially due to the $\mathrm{CM}$ voltage. However, transfer of differential mode (DM) current to CM current may exist. Thus, it is necessary to quantify unbalances in the cable and in the induction machine to apprehend the transfers of modes. Indeed, a transfer of mode is possible only if there is a dissymmetry in the impedance matrix from a CM point of view. In this case, there is a contribution of the differential current to the CM current. To highlight these effects, the following experiments, depicted by Fig. 5 have been done. A balun transformer is used to realize 


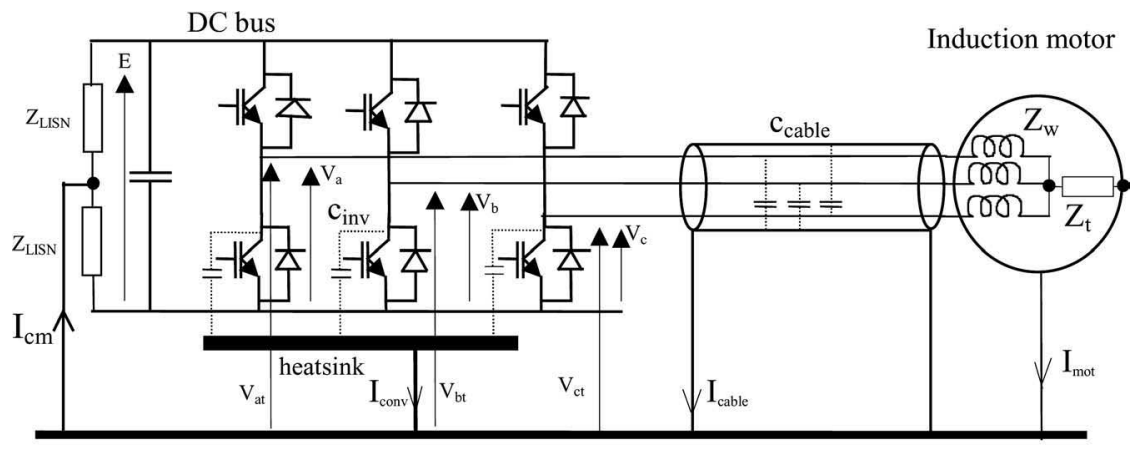

Ground

Fig. 3. Lumped element equivalent circuit of the speed drive system.

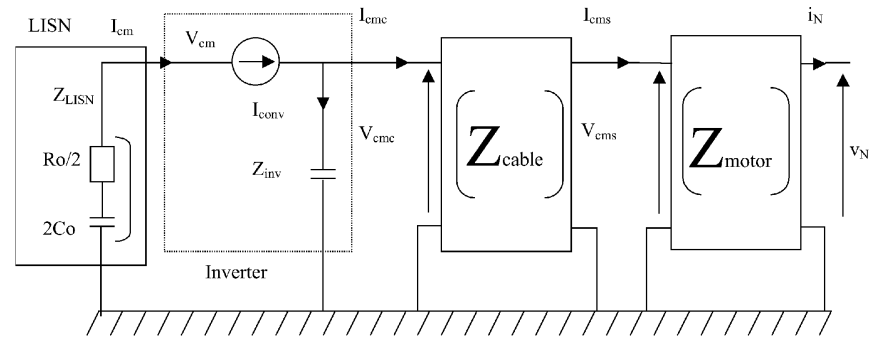

Fig. 4. Equivalent $\mathrm{CM}$ circuit represented by a chain of quadripolar matrices.

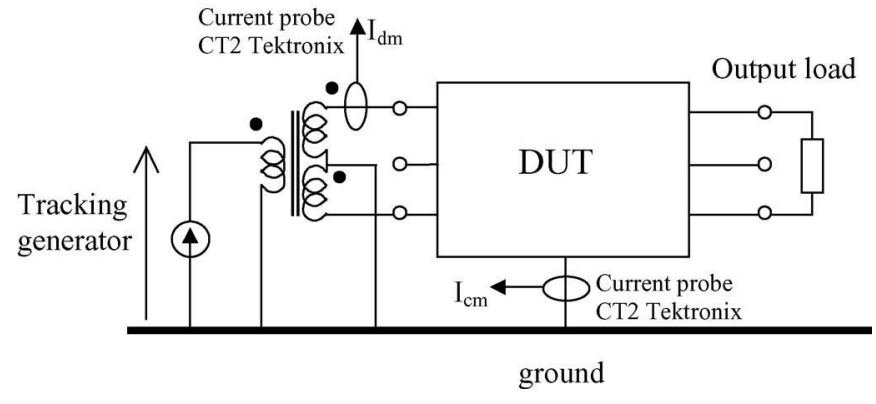

Fig. 5. Principle of the transfer of mode measurements.

a symmetric excitation of the device under test (cable or motor). The principle consists to inject a DM current and to measure what arises in $\mathrm{CM}$. Measurements are made by pairs on the three entries of the device under test (DUT) according to Fig. 5, a load is connected at output ends of the DUT (short circuit, $50 \Omega$, open circuit).

The $I_{\mathrm{cm}} / I_{\mathrm{dm}}$ ratio is measured thanks to a network analyzer (HP4195A) according to frequency. If there is no mode coupling, the ratio tends toward 0 (-infinite in decibels). As a preliminary, the effects of connections and the bandwidth of the current probes have been corrected by the internal compensation of the network analyzer. The results concerning the cable are shown in Fig. 6.

As long as the frequency is lower than $4 \mathrm{MHz}$, whatever the output load, the transfer of mode is lower than $20 \mathrm{~dB}$. Beyond this, there is indeed a transfer of mode. Imbalances are probably due to small differences in the mutual between wires and shielding, and due to weak differences in capacitances with respect to the shielding. The positioning of the cable (lengthened or rolled up on itself) has an effect on the peaks of the function of coupling.

Concerning the motor, two series of measurements were made under the same conditions as the cable. The first series was made similarly as the cable with various ending loads (the more realistic been the short circuit in the case of an induction motor), whereas the second series

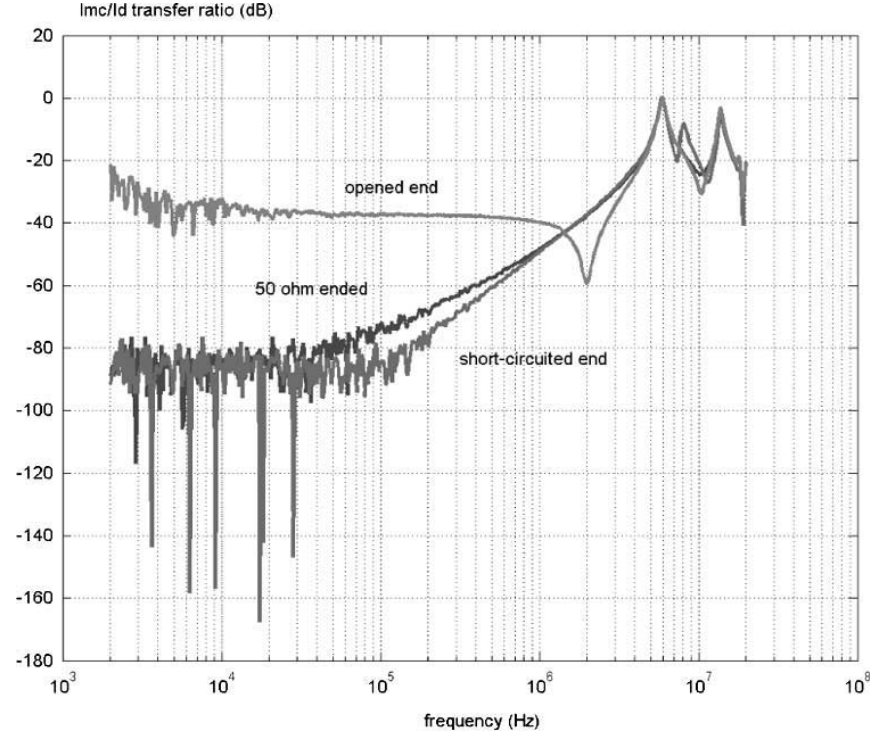

Fig. 6. Transfer ratio DM to CM, shielded cable with four wires of $4 \mathrm{~mm}^{2}$ length: $5 \mathrm{~m}$, according to the ended load.

consisted in measuring the coupling functions per circular shift on the phases, the outputs being loaded by $50 \Omega$.

In the first measurements [Fig. 7(a)], all curves are convergent toward $100 \mathrm{kHz}$, and the transfer of mode remains lower than $-10 \mathrm{~dB}$ until 10 MHz. The second curves [Fig. 7(b)] show that the coupling function depends on the phase winding and that there are rather important differences in the functions of coupling due to inevitable dissymmetry of construction of the induction machine. However, all functions of coupling remain lower than $-10 \mathrm{~dB}$ up to $10 \mathrm{MHz}$. As for the cable, the transfers of mode can become sensitive at high frequency $(>5 \mathrm{MHz})$, but in this case the harmonic content of the DM current is generally negligible because of the filtering effect of the cable and the large motor inductances.

So, in this application we have considered that up to $5 \mathrm{MHz}$, the transfer of DM to CM could be considered negligible; that is the reason why our model considers only $\mathrm{CM}$ effects. Moreover, the $\mathrm{CM}$ source spectrum in our application becomes negligible after $3 \mathrm{MHz}$ [Fig. 10(b)].

However, in other cases where the CM and DM spectra are more spread out, it will be advisable to reconsider this assumption and to wonder about these effects for the disturbances radiated by the shielding of the cables. These elements thus make it possible to explain the good behavior of our CM model. 


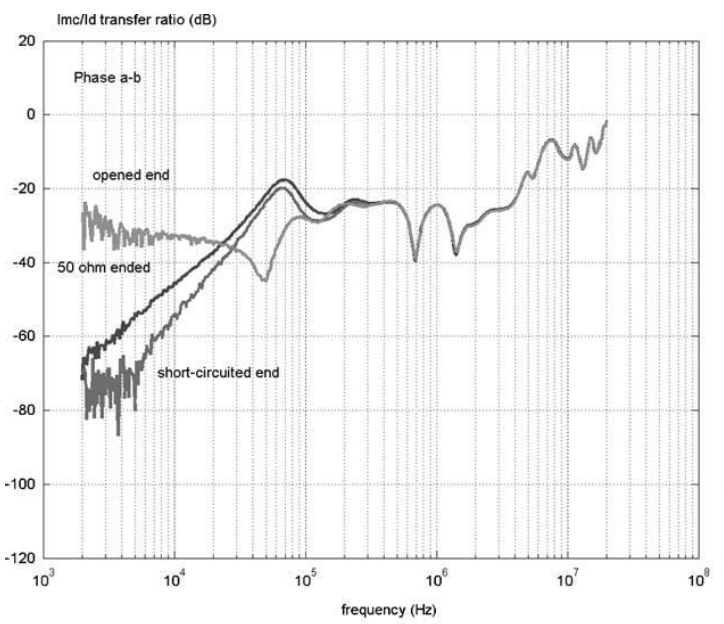

(a)

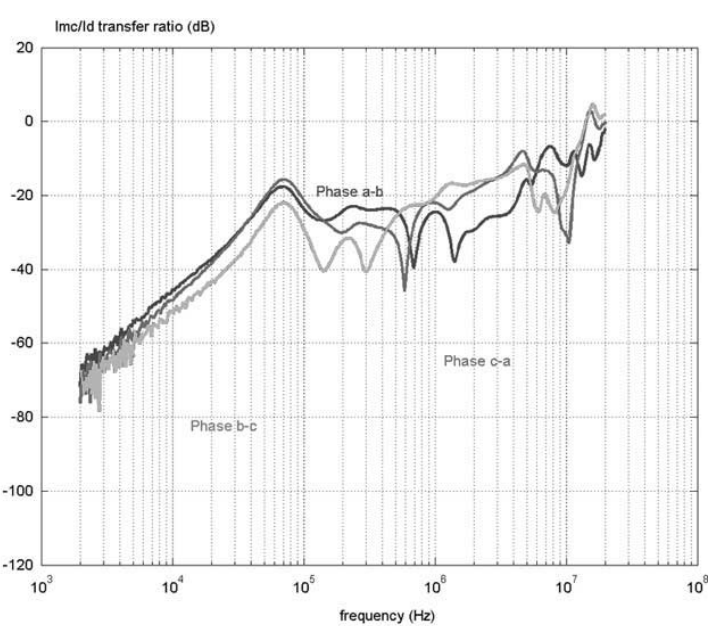

(b)

Fig. 7. (a) Transfer ratio DM to CM as a function of the ended load, Leroy Somer 4-kW motor, $1500 \mathrm{r} / \mathrm{min}$. (b) Transfer ratio DM to CM, permutation of the motor three phases, ending load is $50 \Omega$, Leroy Somer 4-kW motor, $1500 \mathrm{r} / \mathrm{min}$.

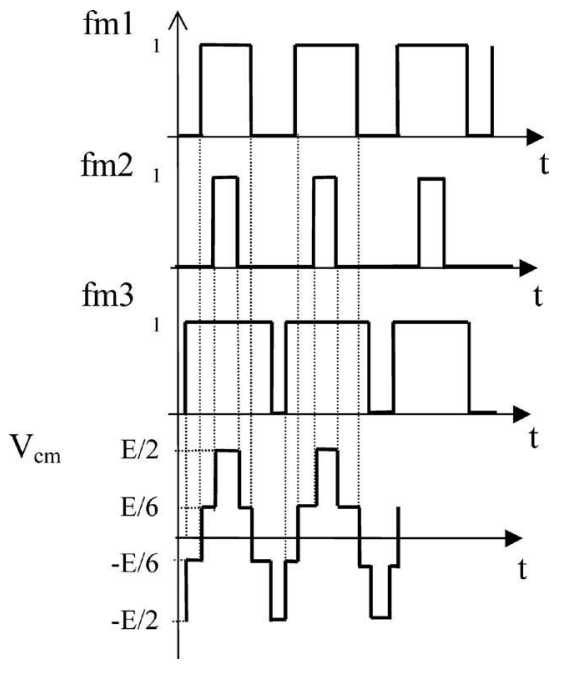

Fig. 8. CM voltage delivered by the inverter.

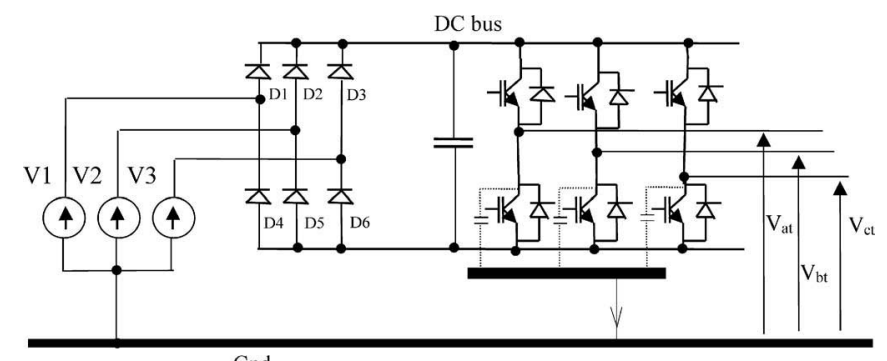

Fig. 9. AC supply of the inverter by a three-phase rectifier.

\section{CM Voltage Identification}

In this section, we show how the CM source is modeled and identified experimentally. The CM voltage $V_{\mathrm{cm}}$ is expressed by [7] (1) (see voltage labels in Fig. 3)

$$
V_{\mathrm{cm}}=1 / 3\left(V_{a t}+V_{b t}+V_{c t}\right)
$$

The system of impedances $Z_{\mathrm{cm}}$ (internal impedances of the LISN) constitutes a voltage divider, which makes it possible to calculate the

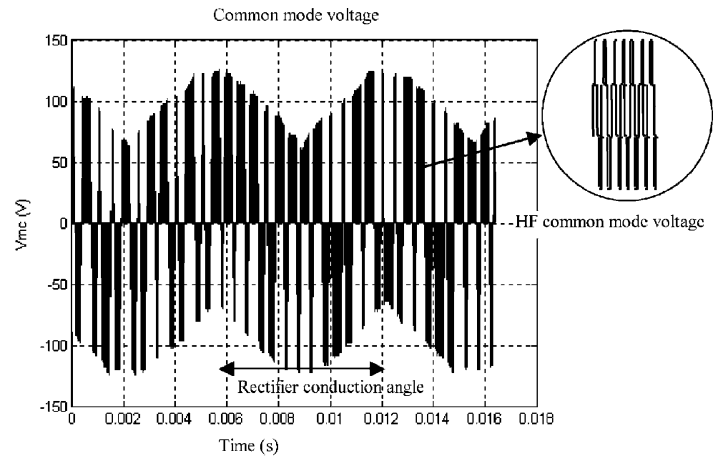

(a)

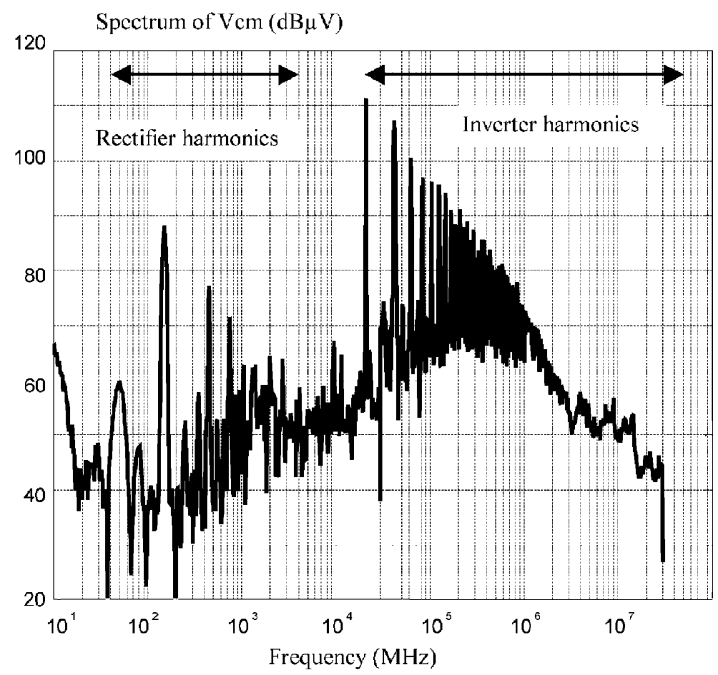

(b)

Fig. 10. (a) $\mathrm{CM}$ voltage modulated by the rectifier working. (b) $\mathrm{CM}$ voltage spectrum.

voltages $V_{a t}, V_{b t}, V_{c t}$, their expressions are given by

$$
\begin{aligned}
& V_{a t}=-E / 2+f m 1(t) \cdot E, \quad V_{b t}=-E / 2+f m 2(t) \cdot E \\
& V_{c t}=-E / 2+f m 3(t) \cdot E
\end{aligned}
$$

where $E$ is the voltage of dc bus of the inverter (Fig. 3). 

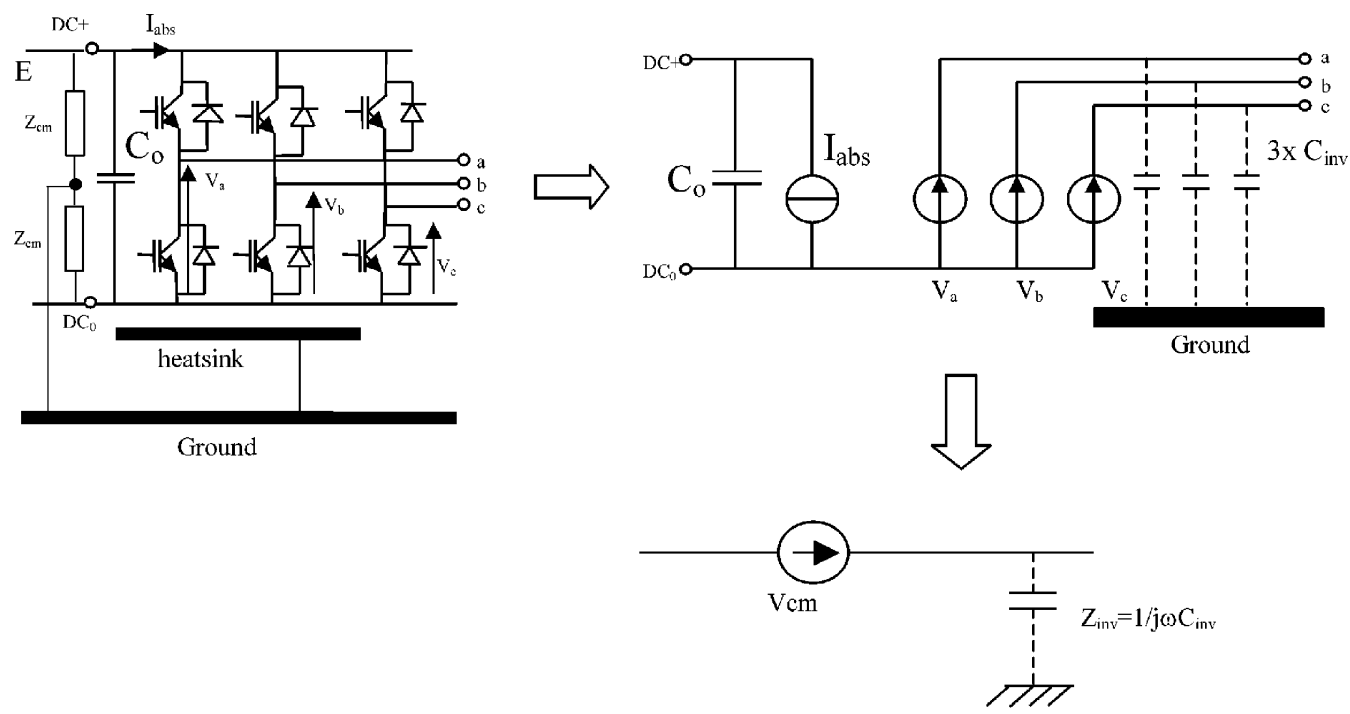

Fig. 11. CM equivalent source of the inverter.

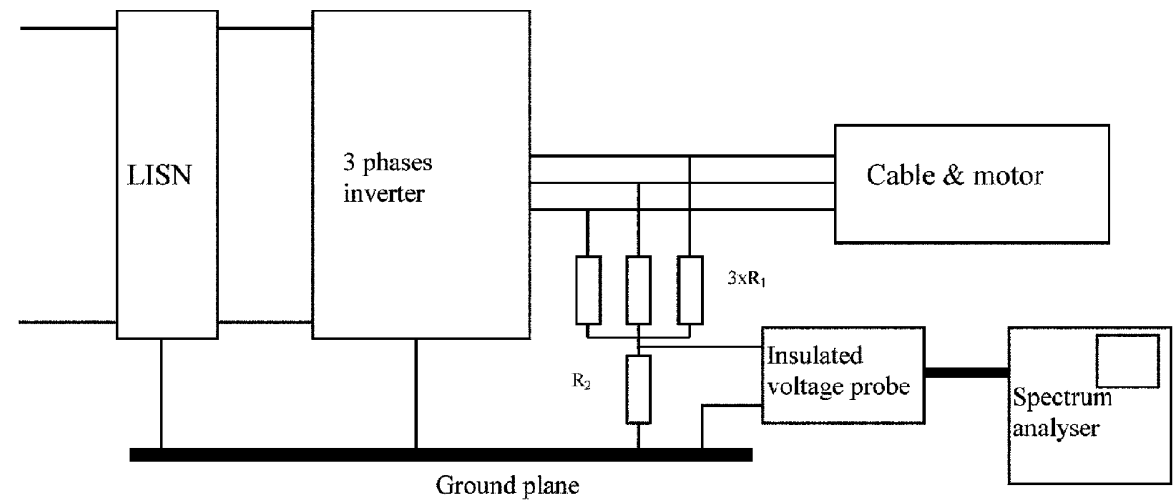

(a)

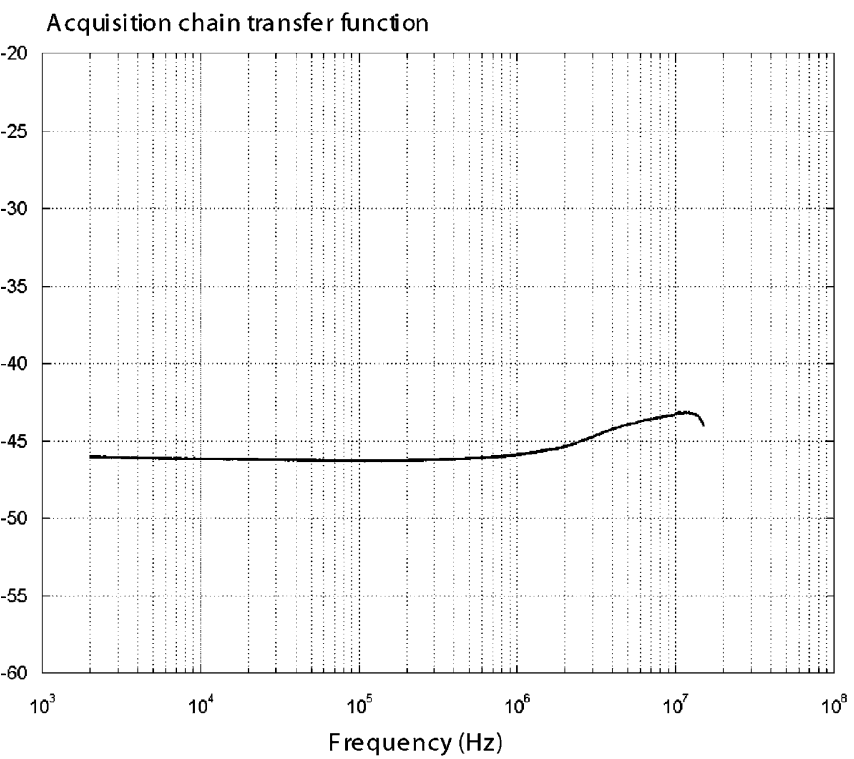

(b)

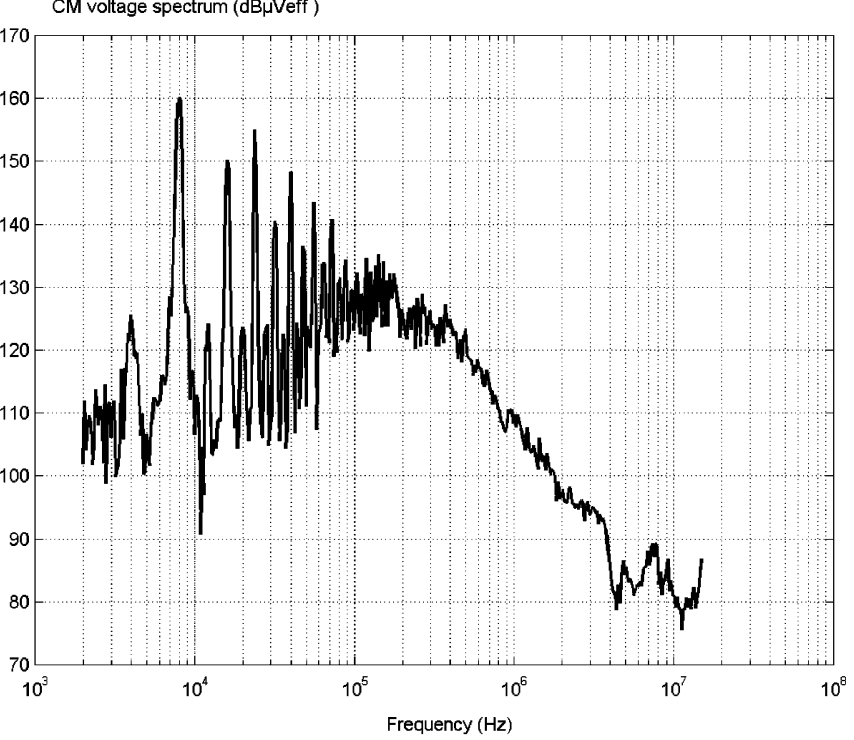

(c)

Fig. 12. (a) CM voltage acquisition chain. (b) Acquisition chain transfer function. (c) Corrected CM voltage spectrum. Analyzer resolution bandwidth: $300 \mathrm{~Hz}$. 
Then

$$
V_{\mathrm{cm}}=[-3 E / 2+(f m 1(t)+f m 2(t)+f m 3(t)) \cdot E] / 3 .
$$

Functions $f m 1, f m 2$, and $f m 3$ are binary functions; they are named "modulation functions" of each leg of the inverter, and they define the output voltages $V_{a}, V_{b}$, and $V_{c}$ of the inverter referenced to the $0 \mathrm{~V}$ of the dc bus (Fig. 3). Assuming a centered PWM, the CM voltage $V_{\mathrm{cm}}$ is represented in Fig. 8. It can be observed that this stairway voltage is particularly perturbing because it is constituted by six transients $(\mathrm{dV} / \mathrm{dt})$ per period, each one injecting a CM current through CM capacitances.

Actually, the dc bus is generally supplied by a three-phase rectifier as represented in Fig. 9.

It can be shown that the rectifier adds a low-frequency term in the expression of $V_{\mathrm{cm}}$. In this case, the new expression of $V_{\mathrm{cm}}$ is given by (4)

$V_{\mathrm{cm}}=1 / 3\{\inf (V 1, V 2, V 3)+[f m 1(t)+f m 2(t)+f m 3(t)] \cdot E\}$

where $\operatorname{Inf}(V 1, V 2, V 3)$ is the minimal value of one of the three grid voltages.

Consequently, the CM voltage contains low-frequency harmonics multiples of $3 \mathrm{~F}_{\text {grid }}(=150 \mathrm{~Hz})$. This effect can be observed in measurements in time and frequency domains in Fig. 10(a) and (b). Measurements have been taken in the industrial variable-speed drive of the test bench.

However, the low-frequency component of the $\mathrm{CM}$ voltage depends largely how the neutral point of the grid is connected to ground.

1) Modeling: The three inverter legs can be considered as perfect ${ }^{1}$ voltage sources as represented in Fig. 11. Similarly, the input current of the inverter $I_{\mathrm{abs}}$ can be considered as a perfect current source.

Finally, using (3), the three voltage sources can be replaced by a single one, $V_{\mathrm{cm}}$, as indicated in Fig. 11. $V_{\mathrm{cm}}$ is expressed by (5)

$$
\begin{aligned}
V_{\mathrm{cm}} & =1 / 3\{-3 E / 2+[f m 1(t)+f m 2(t)+f m 3(t)] \cdot E\} \\
& =-E / 2+1 / 3\left(V_{a}+V_{b}+V_{c}\right) .
\end{aligned}
$$

In this last model, only the CM voltage as been taken into account because DM effects due to $I_{\mathrm{abs}}$ are not considered herein because it has been explained in Section III-B.

An industrial inverter supplied by the three-phase grid $(400 \mathrm{~V} / 50 \mathrm{~Hz})$ has been used in our experiments, its switching frequency was $8 \mathrm{kHz}$, a classical PWM strategy is used to generate the output voltages. The $\mathrm{CM}$ voltage $V_{\mathrm{cm}}$ has been acquired by summation of $V_{a t}, V_{b t}$, and $V_{c t}$ thanks to resistors. It has been directly acquired in frequency domain using a spectrum analyser (HP $4195 \mathrm{~A}$ ) and an insulated voltage probe as presented in Fig. 12(a). The correct working of the inverter required to connect the cable and the motor measurements have been done in this condition. The transfer function of the acquisition chain has been identified to correct the spectrum representation from errors due to the probe bandpass, the resistor network, and the connection fixtures (see response in Fig. 12(b)). Measurements domain extends from the switching frequency $(2 \mathrm{kHz})$ up to the noise-level frequency $(15 \mathrm{MHz})$. Thus, the CM voltage dynamic covers more than four orders of magnitude. Rectifier effects at low frequency have not been considered herein.

\footnotetext{
${ }^{1}$ The impedance of the equivalent source is constituted by the on-state resistance of semiconductors (typically $0.1 \Omega$ ), plus the impedance of the input capacitor of the dc bus (typically, its value is the order of $1000 \mu \mathrm{F}$ with a very low series inductance in the order of $20 \mathrm{nH}$ ), plus the parasitic inductance of the PCB (order of few tenths of a nanohenry). So, the resulting impedance is low and can be neglected for the frequency range of the study $(2 \mathrm{kHz}, 15$ $\mathrm{MHz}$ ). Moreover, this impedance is low compared with the ones connected to the inverter legs.
}

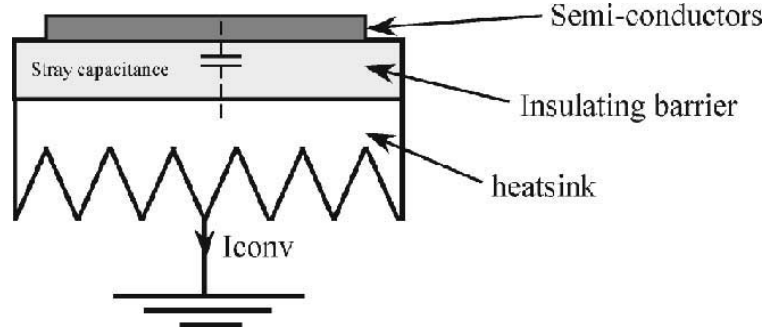

Fig. 13. Capacitive coupling in the power module.

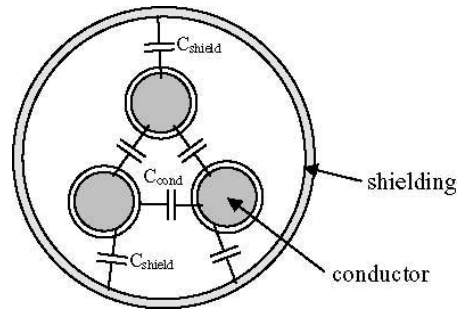

Fig. 14. capacitive couplings in the shielded cable.
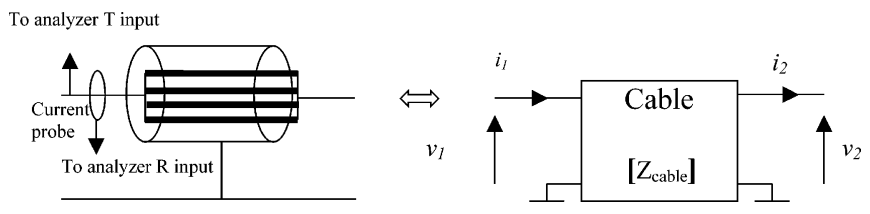

Fig. 15. CM representation of the shielded cable.

Fig. 12(c) shows that the $\mathrm{CM}$ voltage spectrum decreases with a rate of $-20 \mathrm{~dB} / \mathrm{dec}$ up to $300 \mathrm{kHz}$ then $-40 \mathrm{~dB} / \mathrm{dec}$ up to the noise level $(\sim 80 \mathrm{~dB} \mu \mathrm{V})$. Some peaks due to ringings in $V_{\mathrm{cm}}$ can be noticed over $5 \mathrm{MHz}$.

\section{Common Mode Couplings}

This section presents the main capacitive couplings in the system and how they have been modeled. Three main effects have been taken into account.

1) Capacitive Couplings in the Inverter: The capacitive coupling to ground in the converter is mainly due to the power module and its heat sink, as explained by the schematic representation of Fig. 13. As the heat sink is connected directly to ground, this capacitance is a propagation path for the CM current.

The internal CM capacitance $C_{\mathrm{inv}}$ of the inverter described previously has been identified using an impedance analyzer: its value is around $840 \mathrm{pF}$. This is a typical value for this type of speed drive using IPMs (intelligent power modules).

2) Capacitive Coupling in the Cable: To reduce radiated emissions of long cables supplied by high voltage with high $d V / d t$, the inverter output cables are necessarily shielded. The shielding is connected to ground at both ends to ensure an efficient shielding effect. However, this creates capacitive coupling to ground via the capacitances between the conductors and the shielding, as indicated in Fig. 14. This effect is particularly important because the cable is long: the typical value of $C_{\text {shield }}$ is in the order of 30 to $100 \mathrm{pF} / \mathrm{m}$, depending on the cableinsulating materials and its cross-section.

3) Modeling of the Shielded Cable: Because of the complexity to accurately model cables [8] (complex association of twisted copper conductors, dielectric constant and losses not accurately known, inhomogeneous shielding braid) we have adopted a quadripolar representation of a specified length of cable considered in CM (i.e., all 


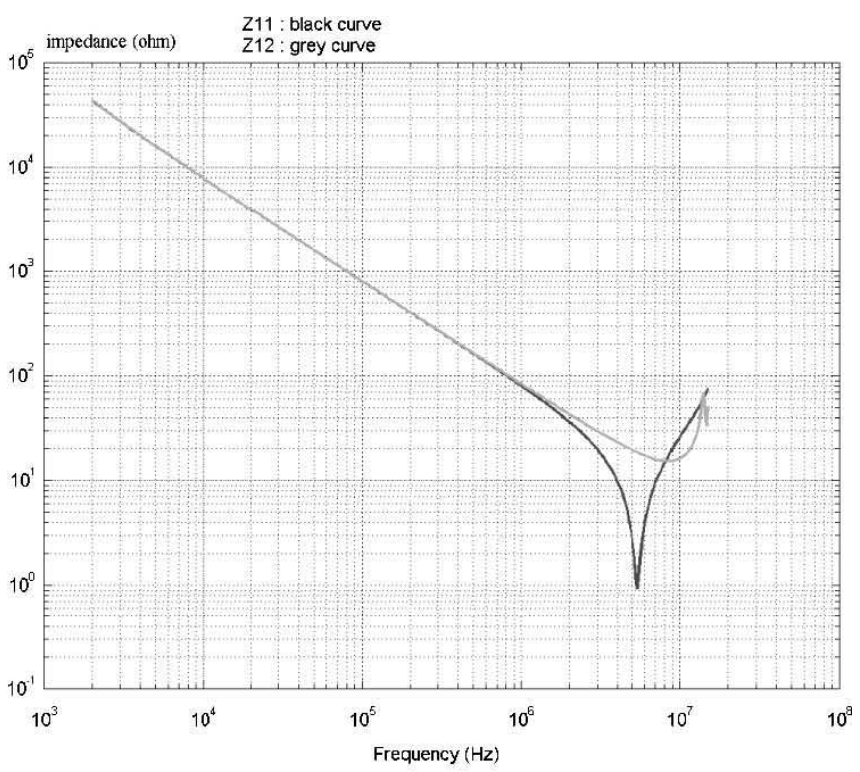

(a)

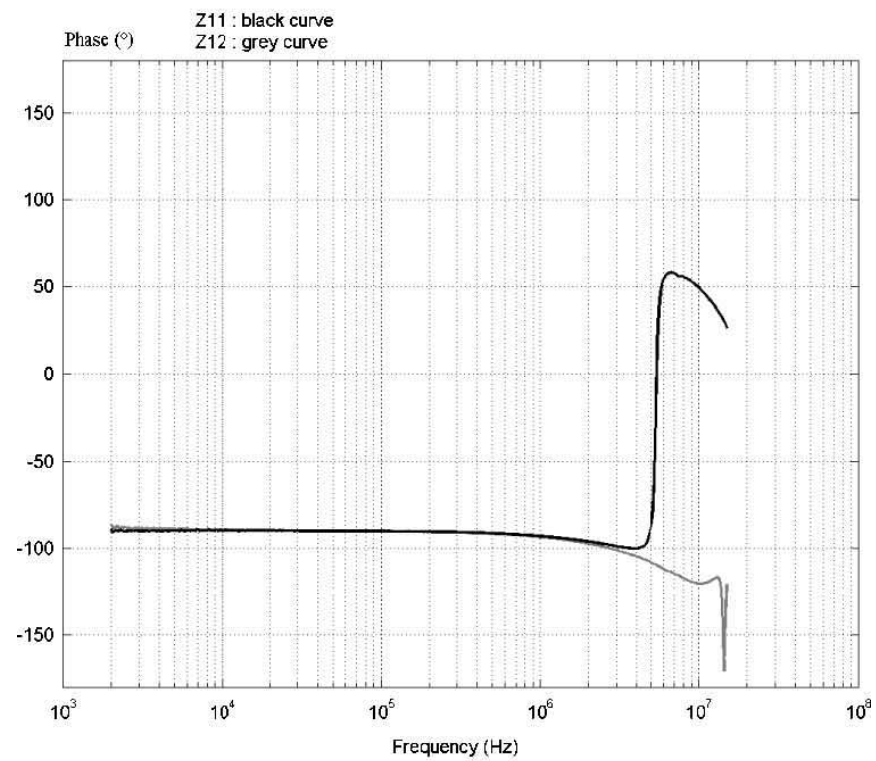

(b)

Fig. 16. Five-meter long $Z_{11}$ and $Z_{12}$ shielded cable CM impedance, modulus (a) and phase (b).

conductors are seen in parallel by the parasitic current). It has been characterized by an impedance matrix $\left[Z_{\text {cable }}\right]$, as presented in Fig. 15. This representation has three advantages:

- simplicity: the coefficients can be acquired by measurements or from a physical model which can be drawn from finite element calculations;

- accuracy, since the model is based on direct measurement;

- versatility, since any cable length can be modelled by the chaining of elementary matrices.

Inversely, the model has to be characterized for any new type of cable, but the database can be easily fed by systematic measurements.

The relationships between input and output current and voltage are expressed by (6)

$$
\left(\begin{array}{c}
v_{1} \\
v_{2}
\end{array}\right)=\left(\begin{array}{ll}
Z_{11 c} & Z_{12 c} \\
Z_{21 c} & Z_{22 c}
\end{array}\right) \cdot\left(\begin{array}{c}
i_{1} \\
-i_{2}
\end{array}\right) .
$$

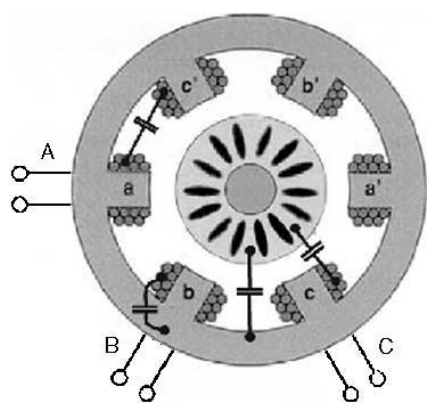

Fig. 17. Representation of the main capacitive couplings in the induction motor.

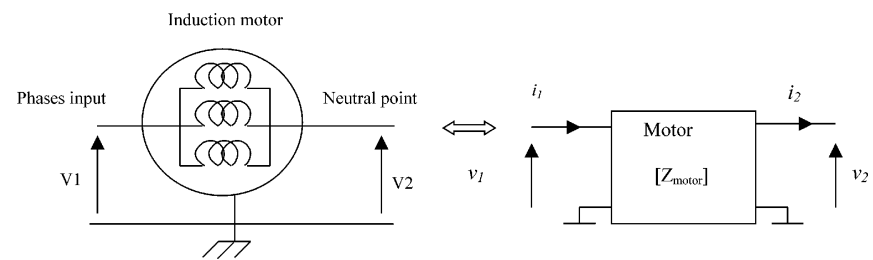

Fig. 18. Induction motor CM representation.

The various impedances can be practically measured in the conditions indicated here:

$$
\begin{aligned}
& Z_{11 c}=\frac{v_{1}}{i_{1}} \quad \text { with } i_{2}=0 \quad \text { and } \\
& Z_{21 c}=\frac{v_{2}}{i_{1}} \quad \text { with } i_{2}=0 \quad \text { (open circuit) } \\
& Z_{12 c}=-\frac{v_{1}}{i_{2}} \quad \text { with } i_{1}=0 \quad \text { and } \\
& Z_{22 c}=-\frac{v_{2}}{i_{2}} \quad \text { with } i_{1}=0 \text { (open circuit) } \\
& Z_{11}=Z_{22}, \quad Z_{12}=Z_{21} .
\end{aligned}
$$

Practically, impedance measurements are achieved using a network analyser and a current probe as presented in Fig. 15: the analyzer indicates the ratio $Z=T / R$. Impedance $Z_{12}$ is obtained by displacing the current probe at the cable output. However, measurements are disturbed by the impedance of the connection fixture (coaxial cables) and by the current probe transfer function. So, initial calibrations have been conducted, and all measurements have been corrected to balance these effects. Fig. 16 shows typical corrected measurements for a $5-\mathrm{m}$ shielded cable. Note that $Z_{i j}$ are not affected by the cable position because they are measured when both ends of the shielding braid are connected to a perfect copper ground plane.

We can notice that the impedances $Z_{11}$ and $Z_{12}$ are identical up to $3 \mathrm{MHz}$; beyond this frequency, inductive effects can be observed and the two curves are branched off.

4) Modeling of the Induction Motor: The schematic representation of a three-phase induction motor is depicted in Fig. 17. Four kinds of capacitive effects can be observed:

- capacitance between the stator winding and the motor chassis;

- capacitance between the stator and the rotor;

- capacitance distributed in the windings of each phase;

- capacitance between the stator winding and the rotor.

These capacitive effects are distributed in the induction motor and their accurate modeling is difficult [5], [6] because of the variety of materials and because of the geometry complexity. 


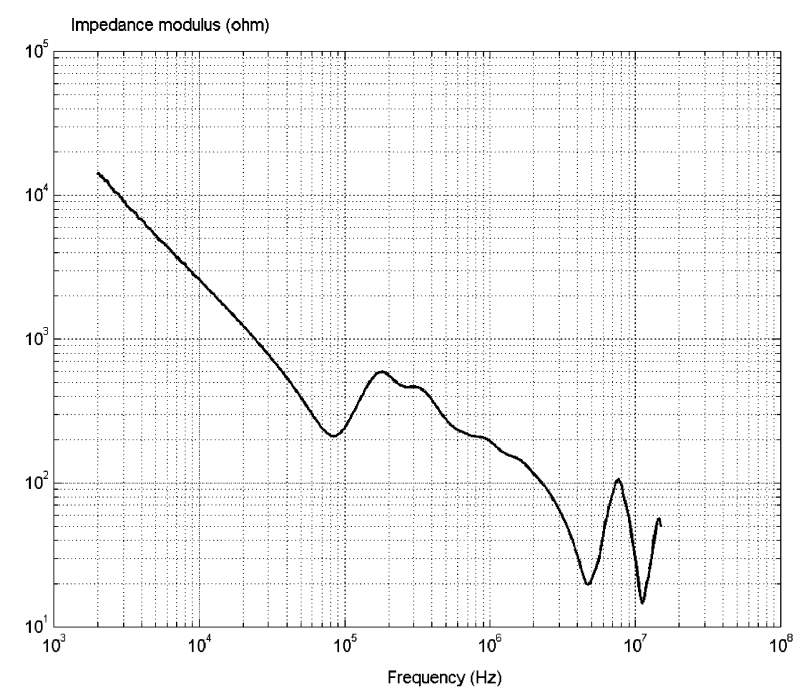

(a)

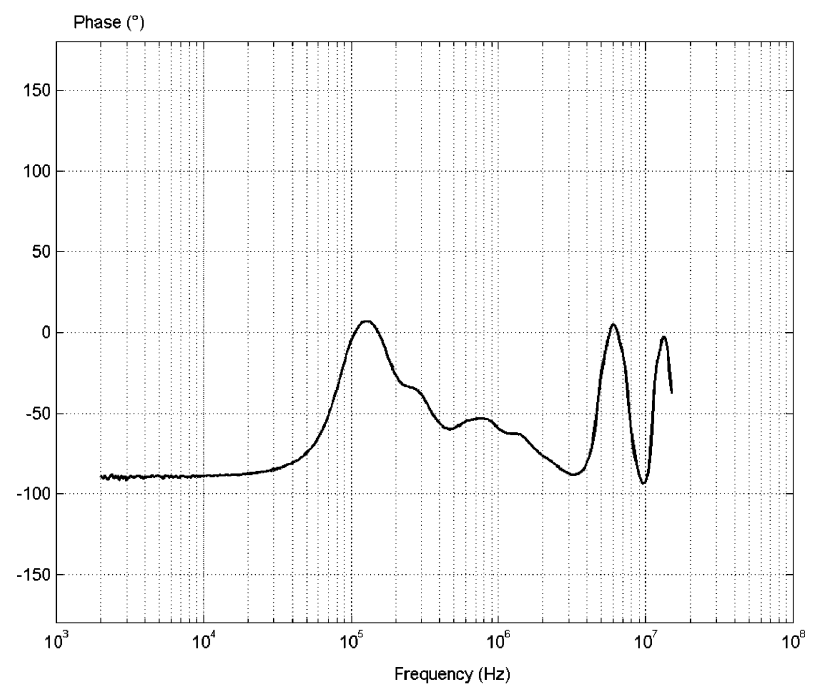

(b)

Fig. 19. $\mathrm{CM} Z_{11}$ impedance of the induction motor, modulus (a) and phase (b).

The modeling principle adopted for the induction motor is the same as for the cable: in CM, the three phases of the motor are considered in parallel and can be represented by a quadripolar $Z$ matrix as represented in Fig. 18.

Measurements have been done in the same conditions as previously explained. Fig. 19 shows the typical CM input impedance $Z_{11}$ of the studied induction motor (Leroy Somer, $1500 \mathrm{r} / \mathrm{min}, 400 \mathrm{~V} / 50 \mathrm{~Hz}$, $3 \mathrm{~kW}$ ).

This curve is globally decreasing attesting a dominant capacitive effect. However, multiple resonance and antiresonance can be observed from $80 \mathrm{kHz}$ to $15 \mathrm{MHz}$, attesting of complex CM and DM scattered effects in the motor windings. This kind of curve explains why our experimental approach has been developed: contrary to the case of the cable, such a curve is quite impossible to obtain with any kind of threedimensional finite element simulator from the dimensions of the motor and from the properties of its materials [6].

To go further, it is possible to consider the rotor shaft as an electrical output where a CM current flows toward the position sensor or toward the driven load. In a similar way, a quadripolar representation can be extracted to determine a specific coupling involving these elements.

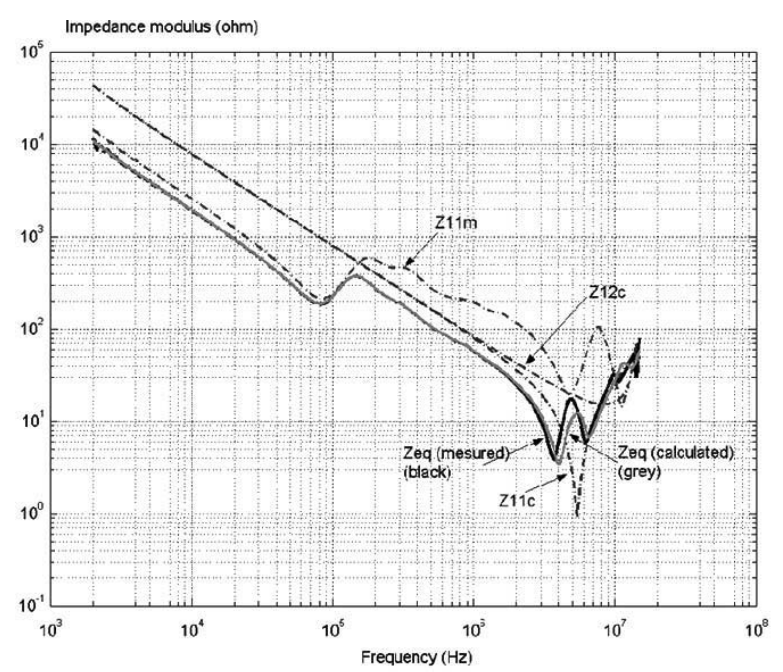

(a)

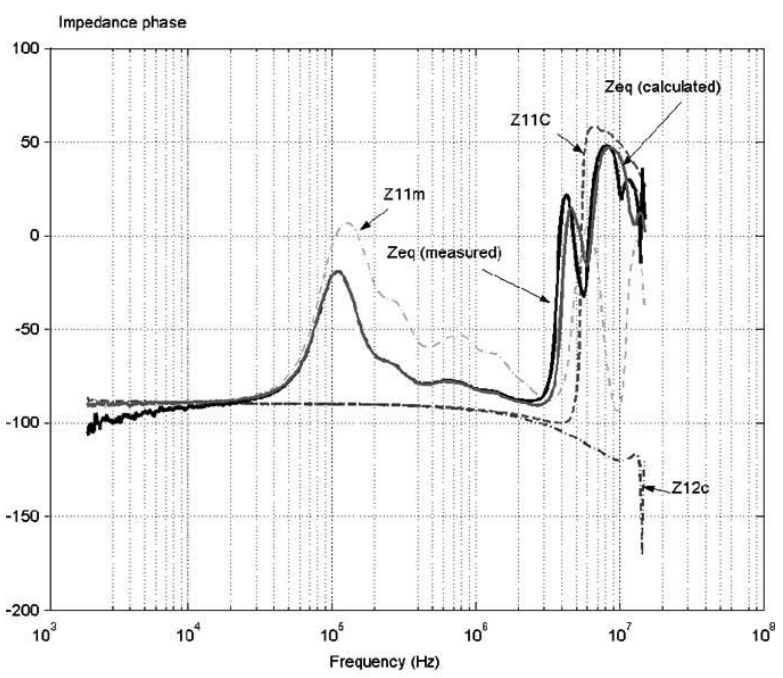

(b)

Fig. 20. Comparison between calculated CM impedance and a direct measurement, modulus (a) and phase (b).

\section{E. Modeling of the Complete System}

1) Association of the Cable and Motor Models: To assess the modeling principle, we have compared the cable + motor CM impedance obtained from direct measurements and calculated from matrices association. This CM impedance is defined by (see notations in Fig. 4)

$$
z_{\mathrm{eq}}=\frac{V_{\mathrm{cmc}}}{I_{\mathrm{cmc}}}=z_{11 c}-\frac{z_{12 c} z_{21 c}}{z_{11 m}+z_{22 c}}
$$

where $Z_{i j c}$ correspond to the cable and $Z_{i j m}$ correspond to the motor.

Fig. 20 presents a comparison between the calculated impedance and a direct measurement. The different impedances used in the calculation have been indicated in the diagram (dotted lines). It can be noticed that the association method of quadripole to describe the CM circuit is efficient; minor differences can be noted around 4 to $5 \mathrm{MHz}$.

2) Complete Calculation of the Common Mode Current: At this step, all impedances being known, it is possible to calculate CM currents in all parts of the system represented in Fig. 3. The expressions of the CM currents in the different locations of the speed drive system 


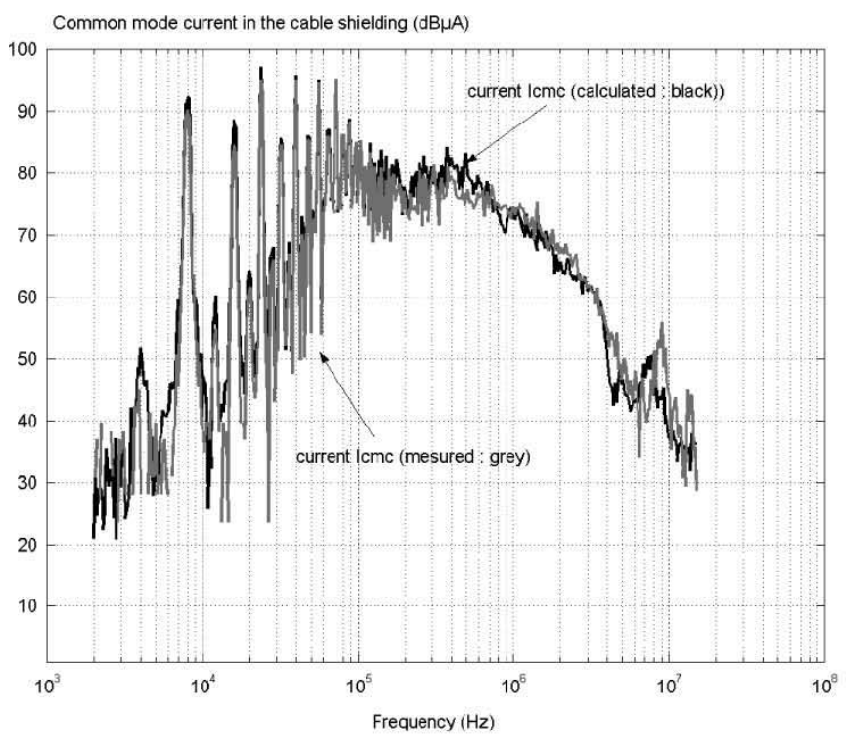

(a)

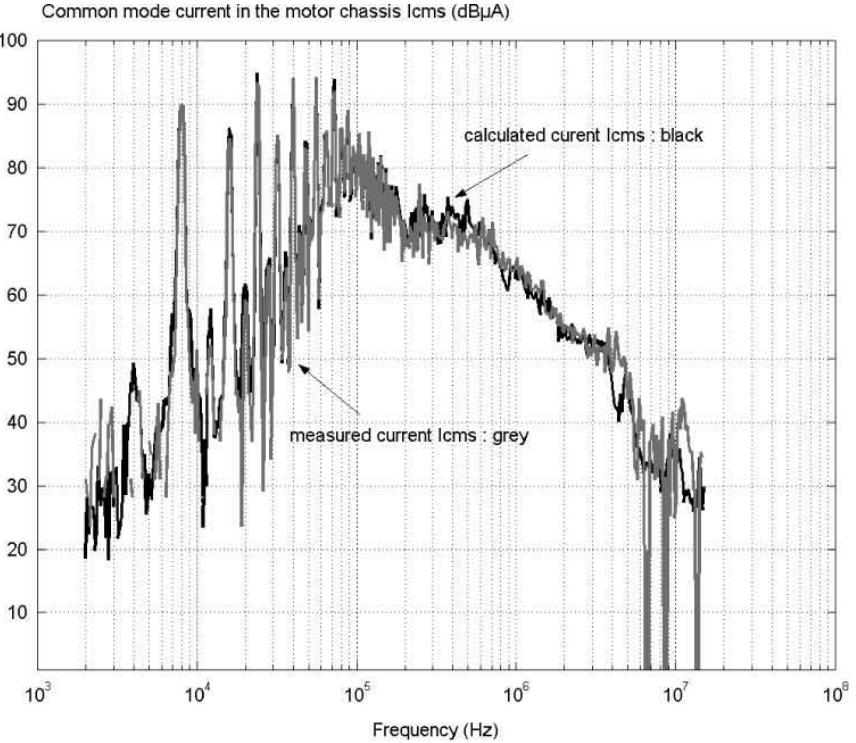

(b)

Common mode current in the LISN Icm (dBHA)

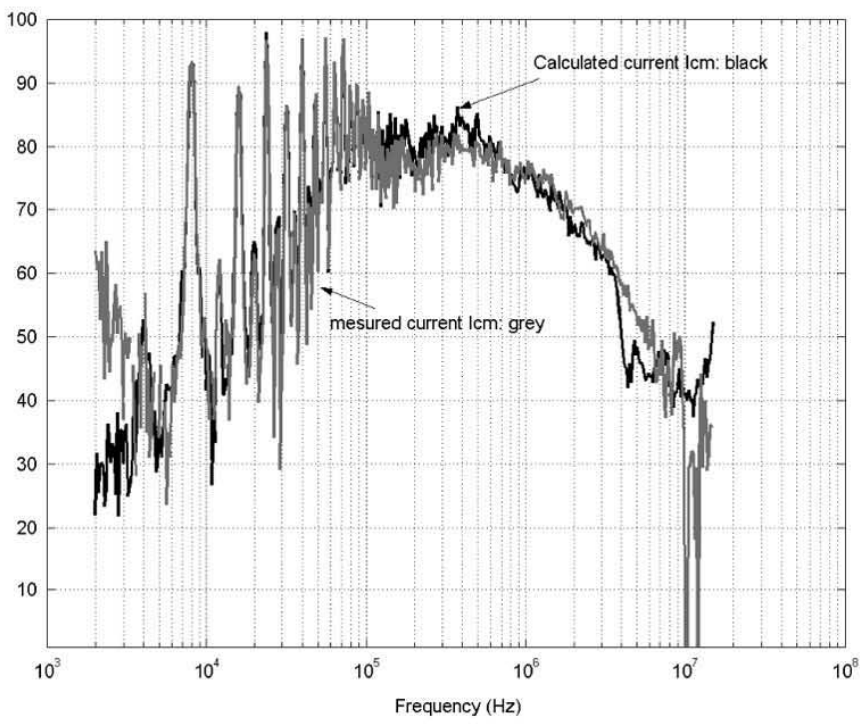

(c)

Fig. 21. Comparison between measured and calculated CM currents in the different parts of the speed drive system. (a) CM current in the cable braid. (b) CM current in the motor chassis. (c) CM current in the LISN.

are given by (10)-(12). These currents can be located in the model of Fig. 4, which suggests how they can be calculated.

The CM current flowing in the LISN is noted $i_{\mathrm{cm}}$, its expression is given by (10), the global impedance seen by the CM source is given by the denominator term

$$
\begin{aligned}
i_{\mathrm{cm}} & =\frac{v_{\mathrm{cm}}}{z_{\mathrm{LISN}}+z_{\mathrm{inv}} / / Z_{\mathrm{eq}}} \\
= & \frac{v_{\mathrm{cm}}}{z_{\mathrm{LISN}}+\frac{z_{\mathrm{inv}}\left(z_{11 c}-\frac{z_{12 c} z_{21 c}}{z_{11 m}+z_{22 c}}\right)}{z_{\mathrm{inv}}+\left(z_{11 c}-\frac{z_{12 c} z_{21 c}}{z_{11 m}+z 2 c}\right)}} .
\end{aligned}
$$

The CM current flowing in the shielding braid of the cable $i_{\mathrm{cmc}}$ is given by (11)

$$
i_{\mathrm{cmc}}=\frac{z_{\mathrm{inv}} v_{\mathrm{cm}}}{z_{\mathrm{LISN}} z_{\mathrm{inv}}+\left(z_{\mathrm{LISN}}+z_{\mathrm{inv}}\right)\left(z_{11 c}-\frac{z_{12 c} z_{21 c}}{z_{11 m}+z_{22 c}}\right)} .
$$

Finally, the CM current flowing in the chassis of the induction motor $i_{\mathrm{cms}}$ is given by (12) at the bottom of the page. The calculation is done in frequency domain: for each harmonic component of $V_{\mathrm{cm}}$, a corresponding current harmonic is calculated using previous formulas. Direct measurements of these currents have been done and compared with calculations as represented in Fig. 21(a)-(c). Current

$$
i_{\mathrm{cms}}=\frac{z_{21 c} z_{\mathrm{inv}} v_{\mathrm{cm}}}{\left(z_{21 m}+z_{22 c}\right)\left(z_{\mathrm{LISN}} z_{\mathrm{inv}}+\left(z_{\mathrm{LISN}}+z_{\mathrm{inv}}\right)\left(z_{11 c}-\frac{z_{12 c} z_{21 c}}{z_{11 m}+z_{22 c}}\right)\right)}
$$


measurements have been conducted using a Tektronix CT2 current probe (bandwidth $=2 \mathrm{kHz}-200 \mathrm{MHz}$ ).

These curves show that our method is particularly efficient in predicting accurately the CM currents in any location of our speed drive system, provided that all impedances and CM sources are accurately determined. Some differences limited to 3 to $5 \mathrm{~dB}$ can be locally observed.

\section{CONCLUSION}

In this paper, we described a pragmatic methodology of predicting the $\mathrm{CM}$ conducted perturbations in a power electronic-based speed drive system, supplied by a three-phase grid and an inverter. We have proposed a modeling technique based on experimental determinations and on a representation of the full system by quadripolar matrices. The model is reduced to the only CM propagation, largely responsible for the major disturbances generated by these devices. We have chosen to minimize the complexity of the model to make it easily programmable; the chaining of quadripole is interesting from this point of view. The results obtained proved that it is possible to accurately determine the amplitude of CM currents in various locations of the system. We have briefly indicated the protocols of measurements required to obtained accurate values of the CM impedances and of the sources. This tool is fully adapted to the designing of filters for the reduction of conduced CM perturbations. Usually, EMI filters are qualified with a $50-\Omega$ load while being fed by a $50-\Omega$ internal impedance generator. The behavior of such a filter in a system where the grid impedance is very different can reveal EMC mismatching. While inserting a CM filter in a modeling tool such as presented herein, it is possible to design its component to obtain the desired attenuation with actual impedances. Last, this architecture of model is sufficiently versatile to be adapted to parameters resulting from physical models, either from simulations or from measurements as carried out herein. Moreover, this method is well matched to represent the CM current flowing in any part of the system: main, inverter, feeding cables, motor chassis, or even rotor shaft or driven load.

\section{REFERENCES}

[1] D. Busse, J. Erdman, R. J. Kerkman, D. Schlegel, and G. Skibinski, "Bearing currents and their relationship to PWM drives," IEEE Trans. Ind. Appl., vol. 12, no. 2, pp. 243-251, Mar./Apr. 1997.

[2] S. Chen, T. A. Lipo, and D. Fitzgerald, "Modeling of motor bearing currents in PWM inverter drives," IEEE Trans. Ind. Appl., vol. 32, no. 6, pp. 1365-1370, Nov./Dec. 1996.

[3] J. M. Erdman, R. J. Kerkman, D. W. Schlegel, and G. L. Skibinski, "Effect of PWM inverters on ac motor bearing currents and shaft voltages," IEEE Trans. Ind. Appl., vol. 32, no. 2, pp. 250-259, Mar./Apr. 1996.

[4] S. Ogasaara and H. Akagi, "Modeling and damping of high-frequency leakage currents in PWM inverter-fed ac motor drive systems," IEEE Trans. Ind. Appl., vol. 30, no. 4, pp. 721-735, Jul./Aug. 1995.

[5] G. Grandi, D. Casadei, and A. Massarini, "High frequency lumped parameter model for AC motor windings," in Proc. EPE 97 Conf., Trondheim, Norway, 1997, pp. 2.578-2.583.

[6] G. Suresh, H. A. Toliyat, D. A. Rendusara, and P. N. Enjeti, "Predicting the transient effects of PWM voltage on the stator windings of random wound induction motor," IEEE Trans. Power Electron., vol. 14, no. 1, pp. 23-30, Jan. 1999.

[7] T. Shimitzu and G. Kimura, "High frequency leakage current reduction based on a common-mode voltage compensation circuit," in Proc. IEEE Power Electronics Specialists Conf. (PESC 96), Baveno, Italy, 1996, pp. 1961-1967.

[8] S. A. Pignari and A. Orlandi, "Long cable effects on conducted emissions levels," IEEE Trans. Electromagn. Campato, vol. 45, no. 1, pp. 43-54, Feb. 2003. 